\title{
La Dialisi Peritoneale: ancora work in progress
}

\section{Claudia Del Corso}

\author{
U.O. Nefrologia e Dialisi, Ospedale Pistoia-Pescia, Azienda USL 3, Pistoia
}

Peritoneal Dialysis: STill work in Progress

Abstract. In the last 4 years the incidence and prevalence of peritoneal dialysis did not increase in Tuscany. The complications of peritoneal catheter, followed by non-clinical reasons, are the main causes of drop-out to hemodialysis. The promotion of the culture of home dialysis and specific policy actions are considered efficient operations to make peritoneal dialysis less marginal.

Conflict of interest: None.

Financial support: None.

Accettato: 20 Settembre 2013

Non possiamo astenerci dal dare un contributo al ricordo di Amedeo De Vecchi, per il quale sicuramente la Dialisi Peritoneale non è stata "marginale" e alla cui cultura scientifica tanti di noi hanno attinto. Come non ricordare quando, alle prime esperienze sul catetere peritoneale e sulle infezioni, mi avventavo sui suoi articoli, che conservo ancora, zeppi di evidenziature e note.

Dopo aver riletto il suo intervento su TN\&D 2001, ecco alcune riflessioni "toscane".

Negli ultimi quattro anni, l'incidenza e la prevalenza della Dialisi Peritoneale è rimasta quasi costante (13-14\%), con immodificata variabilità intraregionale (in sostanza la stessa prevalenza sempre negli stessi Centri). E ancora non tutti propongono questa metodica dialitica, per cui, quando un paziente va a ricoverarsi presso queste strutture, automaticamente gli viene sospesa la DP e passa all'HD. I pazienti provenienti dal trapianto vengono esclusivamente trattati con HD (e questo la dice lunga sull'integrazione delle metodiche dialitiche).

Il drop-out verso l'HD per cause infettive si è significativamente ridotto, mentre le complicanze del catetere peritoneale sono la principale causa di shift verso l'HD. Gran parte dei pazienti $(25 \%)$ sospende la DP non per motivi clinici, ma per problemi socio-assistenziali. Abbiamo, in Dialisi Peritoneale, una popolazione sempre meno autosufficiente, con sempre maggiori richieste assistenziali, che grava quasi esclusivamente sulle famiglie. Nella maggior parte delle Strutture Nefrologiche indagate (70\%), mancano, a livello aziendale, una programmazione e/o un riconoscimento della DP, le cui prestazioni, peraltro, sono spesso codificate in maniera difforme, aspecifica o, addirittura, non sono codificabili. I locali dove viene effettuata la DP non sono adeguatamente identificati e gli ambulatori sono in comune con altre attività.

Abbiamo ritenuto opportuno metterci insieme, medici e infermieri toscani che si occupano di DP, e confrontare le nostre realtà ed esperienze per migliorare la qualità della nostra Dialisi Peritoneale (prevenzione e controllo delle infezioni e team nefrochirurgico per la gestione del catetere peritoneale e delle sue complicanze). Questo richiede un grande impegno per chi già ci "crede", ma la
Dialisi Peritoneale non è una religione! È un trattamento dialitico domiciliare con comprovate indicazioni e vantaggi.

Work in progress: dalla nostra indagine emerge la necessità di una maggiore divulgazione e promozione della cultura della dialisi domiciliare, di agevolazioni per coloro che si prendono in carico la cura della propria malattia e di interventi a livello politico-istituzionale (Assessorati regionali alla Sanità, Direzioni Aziendali), per poter rendere attuabili i programmi di dialisi domiciliare.

\section{Riassunto}

In Toscana l'incidenza e la prevalenza della Dialisi Peritoneale negli ultimi anni sono rimasti stabili a fronte di comprovati vantaggi clinici ed economici. Dati emersi da indagini effettuate nei Centri della regione suggeriscono che azioni di promozione culturale, di divulgazione, di incentivazioni per la dialisi domiciliare e interventi politico-istituzionali potrebbero implementare questa metodica dialitica.

Parole chiave: Dialisi Peritoneale, Drop-out, Divulgazione dialisi domiciliare

Dichiarazione di conflitto di interessi: L'Autore dichiara di non avere conflitto di interessi.

Contributi economici degli autori: L'Autore dichiara di non aver ricevuto sponsorizzazioni economiche per la preparazione dell'articolo.

Indirizzo degli Autori:

Dr.ssa Claudia Del Corso

Dialisi Peritoneale

U.O. Nefrologia e Dialisi

Azienda Usl 3 Pistoia

Via Cesare Battisti 1

51017 Pescia (PT)

c.delcorso@usl3.toscana.it 